\title{
Comparison Between the Experimentally Determined Orientation Dependence of the Near Edge Structure in Electron Energy Loss Spectra from Graphite with Present Theoretical Formulations.
}

\author{
A. C. Y. Liu and N. J. Zaluzec.
}

Electron Microscopy Center, Materials Science Division, Argonne National Laboratory, $9700 \mathrm{~S}$. Cass Av., Argonne, IL, 60439, USA.

Electron Energy Loss Spectroscopy (EELS) in the Transmission Electron Microscope (TEM) is the technique of choice for the chemical fingerprinting of amorphous carbon compounds at high spatial resolution. In this process crystalline graphite is generally used as the $\mathrm{sp}^{2}$ standard. However, the Energy Loss Near Edge Structure (ELNES) from graphite is highly orientation dependent, making its comparison with amorphous materials fraught. Many works prescribe a set of experimental conditions that minimise the orientation dependence, i.e. the "magic angle"[1]. Not only does this throw away valuable resolution, but there are many discrepancies both between theories, and between experiments. Recent measurements using high angular resolution electron channeling electron spectroscopy (HARECES) from the graphite $\mathrm{K}$ edge are mined to provide a continuous data set to compare to theoretical predictions.

Figure 1 describes the experimental procedure. A highly parallel electron beam is tilted under computer control through small angles over a graphite crystal whose c-axis is parallel to the optic axis of the TEM. The K edge EELS spectrum is collected at each angular point using a small onaxis collection aperture, with an angular resolution of $\sim 0.1 \mathrm{mrad}$. In this way an angularly resolved energy loss surface is constructed.[2] To compare this data to more standard experimental situations the background corrected intensity of the surface is integrated to mimic the effect of increasing the collection angle, $\beta$. A series of EELS spectra are obtained as shown in Figure 2 a). Small $\beta$ results in improved momentum resolution and a relatively higher intensity in the $1 s \rightarrow \pi^{*}$ region of the spectrum. The intensities in the $1 s \rightarrow \pi^{*}$ and the $1 s \rightarrow \sigma^{*}$ regions of the spectrum are integrated for each $\beta$ and the relative weight of the $\pi^{*}$ contribution is calculated for comparison with theory.

Theoretical formulations calculate the inelastic differential cross sections for the $1 s \rightarrow \pi *$ transition and the $1 s \rightarrow \sigma *$ transition, resulting in matrix elements weighted by a function that depends on the convergence and collection angles and also the angle between the c-axis of the crystal and the optic axis. The relative weight of the $\pi^{*}$ contribution may be readily calculated from these weighting functions. We have input our experimental parameters to the theory of Souche and compared experiment to theory, as seen in Figure 2 b).[3] The theoretical value of the relative weight of the $\pi^{*}$ contribution with increasing collection angle is seen to be approximately linear with experiment at low collection angles. However, the data displays a shoulder that is not seen in the theoretical curves at $\beta / \theta_{E} \sim 4$ where $\theta_{E}=\Delta E /\left(\gamma m_{0} v^{2}\right) ; \Delta E$ is the energy loss, $\gamma=1 / \sqrt{1-v^{2} / c^{2}}, v$ is the incident electron velocity, $c$ is the speed of light and $m_{0}$ is the rest mass of the electron. Further investigation and comparison with other theories [4] may be required to understand the source of the discrepancy.

References

[1] N. K. Menon and J. Yuan, Ultramicroscopy, 74, (1998), 83. 
[2] N. J. Zaluzec, etal, Microscopy and Microanalysis, 11, (Suppl 2), (2005) 718.

[3] C. Souche, B. Jouffrey, G. Hug and M. Nelhiebel, Micron, 29, (1998) 419.

[4] B. Jouffrey, P. Schattschneider and C. Hebert, Ultramicroscopy, 102, (2004), 61.

[5] This work was supported in part by the U.S. DoE under BES-MS W-31-109-Eng -38 at ANL

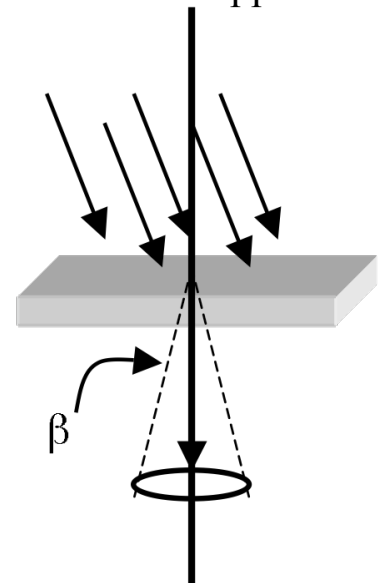

$\alpha=$ convergence angle $\approx 0$

$\gamma=$ angle between c-axis and optic axis $\approx 0$

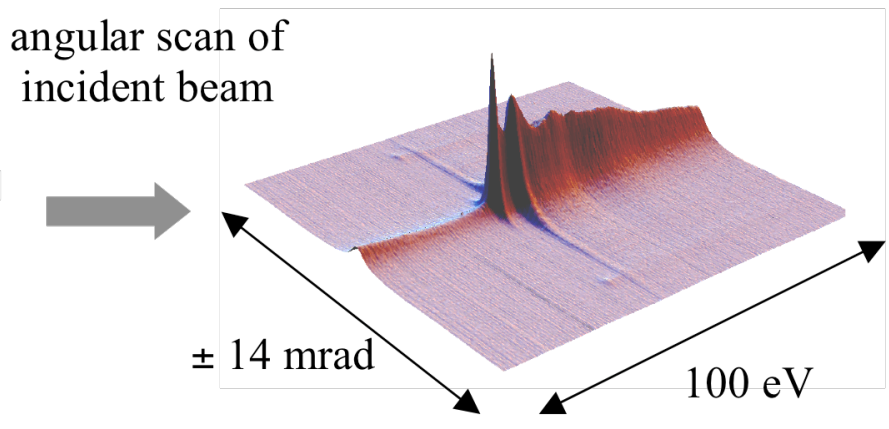

angularly resolved energy loss surface

Fig. 1. Experimental conditions and resultant angularly resolved energy loss surface. The incident illumination is parallel and the angle between the c-axis of the graphite sample and the optic axis is nearly zero.

a)

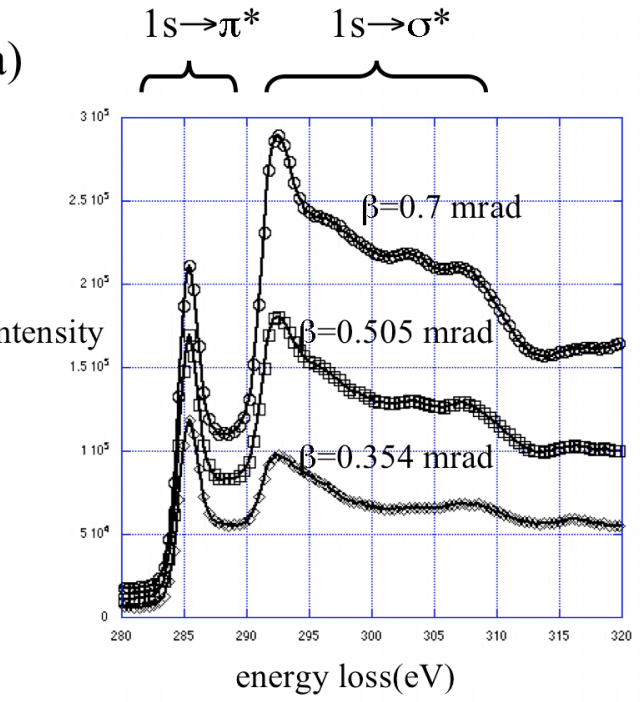

b)

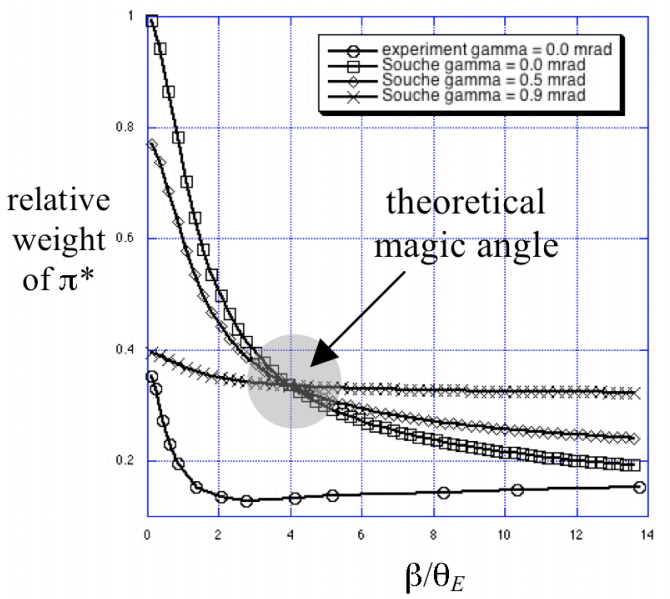

Fig. 2. a) The intensity from the angularly resolved energy loss surface is integrated to examine the effect of varying $\beta$, the collection angle. Small $\beta$ results in enhanced momentum resolution and an enhanced $1 s \rightarrow \pi^{*}$ peak intensity. The relative weight of the $\pi^{*}$ contribution is calculated by integrating intensities from the appropriate regions of the spectra. b) Comparison of the experimental relative weight of the $\pi^{*}$ contribution to the theoretically calculated value for a range of collection angle, $\beta$. $\beta$ is scaled by the characteristic angle. Theoretical plots for three different values of $\gamma$, the angle between the c-axis of the graphite and the optic axis, are also shown. From such a treatment, the "magic angle" can be determined to be $4 \theta_{E}$. 